\title{
ENVIRONMENTAL CONSTRAINTS IN THE EVOLUTION OF SCALAR CONCEPTS: ROAD TO MOST
}

\author{
DARIUSZ KALOCIŃSKI \\ Corresponding Author: d.kalocinski@uw.edu.pl \\ Institute of Philosophy, University of Warsaw, Warsaw, Poland
}

\begin{abstract}
One of the driving forces of language evolution is the selection of variants that suit the communicative needs of its users. Crucially, fitness of linguistic variants may largely depend on the structure of the environment in which language is learned, transmitted, and used. This hypothesis has gained support in various domains. We apply it in the context of scalar terms with a major focus on quantifiers, such as most. Based on a model that combines logic and evolutionary game theory, we argue that such signals might have evolved as stable semantic units through adaptation to general communicative principles and distributional properties of the environment such as normality.
\end{abstract}

\section{Introduction}

During the development across various timescales, languages tend to adopt variants exhibiting greater communicative fitness (Christiansen \& Chater, 2016a). Crucially, the fitness of linguistic variants may largely depend on the structure of the environment in which language is learned, transmitted, and used. This theoretical stance has gained support in several domains, including spatial descriptions (Levinson, 1996, 2003), color categories (Lindsey \& Brown, 2002; Plewczyński et al., 2014), kinship terms (Kemp \& Regier, 2012), and constituent order (Christensen, Fusaroli, \& Tylén, 2016). Quantifiers, despite their ubiquity in natural language, are less explored from this perspective, with notable exceptions focusing on fuzziness and context-dependence (Pauw \& Hilferty, 2012, 2016).

The present paper is an attempt to model the influence of environmental constraints on the evolution of scalar terms, and of proportional quantifiers in particular. Most is a paradigmatic example of such a quantifier: it stands out in terms of frequency in natural language corpora (Szymanik \& Thorne, 2017) and is present across many languages (Katsos et al., 2016). Our goal is to provide some reasons that most might have acquired and sustained its present semantic threshold, commonly associated with $1 / 2$ (Peters \& Westerståhl, 2006). Interestingly, our argument heavily relies on the analysis of a much broader class of scalar concepts.

We briefly describe the notion of the scalar concept in Section 2. Section 3 describes a communication game based on the discrimination of two contexts. In 
Section 4, we analyse those properties of scalar terms that are important when considering their evolution. We present our main argument in Section 5 and the conclusion in Section. 6.

\section{Scalar Concepts}

A scalar concept is represented as a threshold dividing a given range of values. This is easily modelled, for example, as a real/rational number dividing an (extended) interval of real/rational numbers. We do not consider the direction of monotonicity here: we assume that a scalar concept comprises all values exceeding the corresponding threshold.

Tentative examples of scalar concepts are tall and most. Tall may be referred to as a first-order concept, as possible thresholds are properties of individual objects, namely different values of height. Most may be referred to as a second-order concept, as possible thresholds are not properties of individual objects, but rather properties of sets of individual objects (Barwise \& Cooper, 1981). In this particular case, the relevant property is identified with a fraction of cardinalities between corresponding sets. Recall that a typical context for the statement Most As are B consists of two finite sets $A, B$. The standard meaning of most is as follows: Most As are $B$ is true iff $|A \cap B|>|B-A|$, equivalently $|A \cap B| /|B|>1 / 2$. Substituting other proportions for $1 / 2$ yields other proportional quantifier meanings.

\section{Discrimination Game}

To put scalar terms into a communicative setting, consider agents using one shared signal, optionally marked with negation. At any given time, an agent associates one particular threshold with the signal, but different agents may associate it with different thresholds. Suppose we have an agent using threshold $p$ and his interlocutor using $q$. They want to discriminate between two (shared) contexts $c$ and $c^{\prime}{ }^{1}$ Such a game is a success iff both players can tell the difference between the contexts using their current strategies. ${ }^{2}$ Formally, players receive the payoff 1 iff $\min \left(c, c^{\prime}\right) \leq \min (p, q) \leq \max (p, q)<\max \left(c, c^{\prime}\right)$. Otherwise, the payoff is 0 .

The variability of contexts is modelled by a random variable $X$ (environment). Each time a game is to be played, two contexts are drawn from $X$. When two players meet and they happen to use strategies $p$ and $q$, the likelihood that they are both capable of discriminating between upcoming contexts is

$$
D_{X, p, q}=2 P(X \leq \min (p, q)) P(X>\max (p, q)) \text {. }
$$

\footnotetext{
${ }^{1}$ We use the term "context" in a different way then does Steels (1997). It it is more natural to speak about a quantified sentence evaluated against a context (rather than against object or event happening in a shared context).

${ }^{2}$ If at least one of the players cannot do this using his current strategy, we view this as a failure: the sender has no incentive to use such a (non-discriminative) signal, while the receiver will not understand which of the contexts is the topic. Our game is a simplified version of that considered in Pauw and Hilferty (2012).
} 
which are the chances of obtaining a pair of random deviates $\left(c, c^{\prime}\right)$ satisfying $p \in\left[c, c^{\prime}\right)$ and $q \in\left[c, c^{\prime}\right)$. Observe that, when $p=q$, we have $D_{X, p, q}=$ $2 P(X \leq p) P(X>p)$. We will use $D_{X, r}$ to denote the value of $D_{X, r, r}$.

\section{Look at Evolution}

We want to compare various strategies with regard to their communicative fitness under plausible environmental constraints. Our chief assumption about the environment is normality, i.e. $X \sim \mathcal{N}\left(\mu, \sigma^{2}\right)$, for some $\mu \in \mathbb{R}$ and $\sigma>0$. This is a tentative but fairly realistic assumption, as many properties are known to behave in this way. Our analysis is based on standard tools of evolutionary game theory. ${ }^{3}$

We consider a large population of agents who are paired randomly to play the discrimination game. For simplicity, we confine our attention to two co-existing strategies, $p$ and $q$. Let $x_{p}=1-x$ and $x_{q}=x$ be the fractions of the population using strategies $p$ and $q$, respectively. The communicative fitness of a $p$ and a $q$-individual, given in Equations $2 \mathrm{a}$ and $2 \mathrm{~b}$, is expressed as the expected payoff she receives from a discrimination game while being randomly paired with other agents (the chances of meeting someone endorsing $p$ and $q$ are $1-x$ and $x$, respectively):

$$
\begin{aligned}
& E_{p}(x, X)=(1-x) D_{X, p}+x D_{X, p, q} \\
& E_{q}(x, X)=(1-x) D_{X, p, q}+x D_{X, q}
\end{aligned}
$$

Given strategies $p, q$ and a random variable $X, p$ is said to be evolutionarily stable against $q$ in $X$ if there is $y, 0<y \leq 1$, such that for all $x<y, E_{p}(x, X)>$ $E_{q}(x, X)$. We say that $p$ is immune to invasion in $X$ if, for all strategies $q$ such that $q \neq p, p$ is evolutionarily stable against $q$ in $X$.

It is not difficult to see that every strategy is immune to invasion in $X$. Hence, once a strategy has dominated the entire population, it cannot be replaced by any other strategy through the invasion of an initially very small number of mutants.

Strategies, although immune to invasion, are not equivalent in terms of their expected payoffs. A crucial property of two (different) strategies $p, q$ is the population threshold $x_{0}$ at which $E_{p}\left(x_{0}, X\right)=E_{q}\left(x_{0}, X\right)$. A slight modification in the proportions of strategies within a population, $x_{0} \pm \varepsilon$, provides an advantage to one of the strategies in terms of communicative fitness.

Figure 1 shows how different strategies are related to each other with regard to population thresholds in the standard normal environment. Observe that an invading strategy $q$ can outperform $p=0$ only at levels $x_{q}>0.5$, in other words, when invaders comprise more than half of the population. However, when $p \neq 0$, strategies $q$, which are closer to the mean than $p$, can outperform it at lower levels,

\footnotetext{
${ }^{3}$ See, for example, Easley and Kleinberg (2010).

${ }^{4}$ It follows from the linearity of the expected payoff, and from the fact that, for all $p, q \in \mathbb{R}$ such that $p \neq q, E_{p}(0, X)=D_{X, p}>D_{X, p, q}=E_{q}(0, X)$.
} 


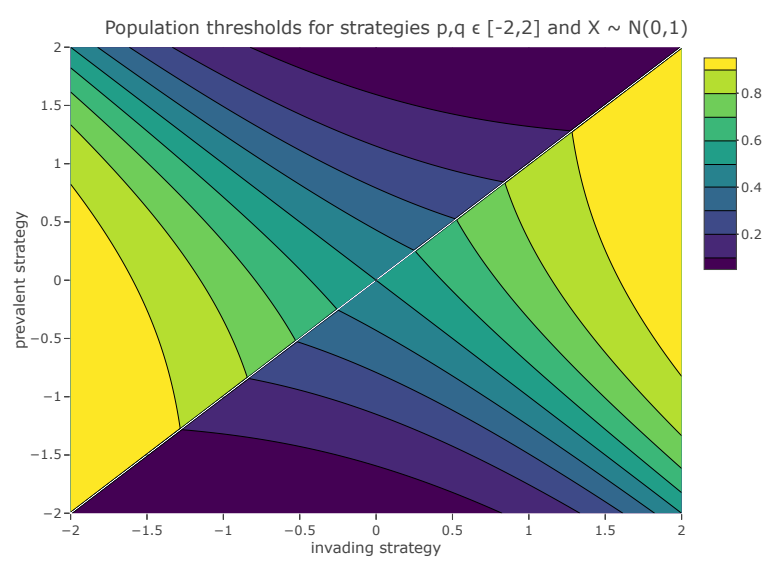

Figure 1. Population thresholds $f(p, q)$ for pairs of strategies $(p, q)$ in the environment $X \sim \mathcal{N}(0,1)$ where $f(p, q)=y \Leftrightarrow E_{p}(y, X)=E_{q}(y, X)$, for all $p, q \in \mathbb{R}$. If $x_{q}>f(p, q), q$ outperforms $p$ in terms of communicative fitness. If $x_{q}<f(p, q)$, the opposite is true.

indicating that even if the invaders are in the minority, they can still spread across the novel semantic threshold successfully. It is interesting that, if strategy $q$ is just a little closer to the mean than is $p$, the population threshold drops sharply and attains very small values for $p$ s lying further from the mean, indicating that overcoming poor conventions should be relatively easy. However, the closer the prevalent strategy is to the mean, the higher the number of invaders required to compromise its communicative fitness. A major lesson is that closeness to the mean is crucial for immunity as understood in terms of population thresholds. Most importantly, the strategy corresponding to the mean is the most advantageous one in this respect.

By employing replicator dynamics (Hofbauer \& Sigmund, 1998), we can obtain insight into how proportions of strategies in a population may change over time. A slight modification to the population threshold for which the rate of growth $\dot{x}_{p}$ of the $p$-subpopulation equals 0 will lead to the total domination of one strategy. These dynamic variants of population thresholds behave roughly the same as do the ordinary population thresholds described above (see supplementary materials).

\section{Road to Most}

A typical context for a proportionally quantified sentence (Most boys are tall) consists of two sets $A, B$ (the set of boys and the set of tall people, respectively). On many occasions, as in our example, $B$ consists of instances of a scalar concept. Based on our previous considerations, assume that first-order scalar concepts have 
developed into thresholds equal to the means of the corresponding normally distributed variables. Let $\mu$ be a concept used for the classification of objects with regard to the property $X \sim \mathcal{N}\left(\mu, \sigma^{2}\right)$. Trivially, the probability $P(X>\mu)$ of judging a random deviate of $X$ as member of the concept $\mu$ is equal to $1 / 2$.

As in the case of first-order scalar concepts, the variability of contexts is modelled probabilistically. Given a set $A$ consisting of $n$ objects, we envisage that $A \cap B$ is obtained from the sequence $X_{1}, X_{2}, \ldots, X_{n}$ of $n$ independent, identically distributed random variables, $X_{i} \sim \mathcal{N}\left(\mu, \sigma^{2}\right), i=1,2, \ldots, n$. Hence, for an $i$ th object of the set $A, P\left(X_{i}>\mu\right)=1 / 2$. As a result, $|A \cap B|$ is the number of successes of $n$ independent Bernoulli trials with the probability of success $1 / 2$, $Y_{n} \sim B(n ; 1 / 2) .{ }^{5}$ For simplicity, we do not mention $A$ at all, and assume that each context consists of $n$ objects in total with the number of objects belonging to $B$ governed by $Y_{n} \sim B(n ; 1 / 2)$. However, what interests us most is the random variable $Y_{n} / n$. It turns out that the mean of $Y_{n} / n$ is $1 / 2$. Based on the normal approximation to binomial distribution, $\mathcal{N}(E[Y / n], \operatorname{Var}(Y / n))$ may be treated as a good approximation to $Y_{n} / n$, provided $n$ is sufficiently large (a liberal rule of thumb is $n p \geq 5$ which, for $p=1 / 2$, yields $n \geq 10$ ).

Given a normal approximation $Z$ to $Y_{n} / n$, the analyses of previous sections applies to proportional quantifiers. Strategies are proportions from [0, 1]. Strategy $1 / 2$ corresponds to most. In general, the truth conditions corresponding to a strategy $r \in[0,1]$ are given by $|A \cap B| /|A|>r$. Hence, the probability that a given context $A, B$ is classified as true with regard to the threshold $r$ is $P\left(Y_{n} / n>r\right)$ which, in turn, is approximated by $P(Z>r)$. All conclusions obtained in previous sections thus remain valid for proportional quantifier strategies, and $1 / 2$ is thus the most advantageous strategy, both in terms of communicative fitness and immunity to invasion. It can also easily invade strategies that are far from $1 / 2$.

\section{Conclusions and Perspectives}

We have argued that the meaning of most, the proportional quantifier occurring so often across so many languages, may be viewed as an adaptation of language to general communicative principles and distributional properties of the environment. Our explanation begins with a normality assumption concerning the properties of individual objects. We show that scalar concepts, when used to refer to such properties, are likely to develop, and sustain, thresholds close the corresponding means. Next, we extend our argument to proportional quantifiers. Assuming the most likely thresholds of first-order scalar concepts, we investigate the probabilistic behaviour of higher-order scalar properties, such as $|A \cap B| /|A|$, which are

\footnotetext{
${ }^{5}$ Obviously, to obtain a full probabilistic model for generating sets $A$ and $B$, we should also make some assumptions about $A$. However, as long as the variables used to obtain $A$ and $B$ are independent, our approach remains valid. We can imagine this in the following way: say our context consists of $k$ objects in total. One variable determines which of the $k$ objects belong to $A$. The other one selects, independently, which of the objects in $A$ have also the property $B$.
} 
crucial in comparisons with threshold proportions of proportional quantifiers. We show that, when $B$ corresponds to a first-order scalar concept, the probabilistic behaviour of $|A \cap B| /|A|$ is approximately normally distributed with a mean of $1 / 2$. This yields precisely $1 / 2$ as the most favourable threshold for a proportional quantifier - a result that aligns well with the usual interpretations of most.

The present findings contribute to theories according to which languages, during their development across various timescales, are driven towards variants exhibiting greater communicative fitness (Christiansen \& Chater, 2016a). Our results reveal that proportional quantifiers are no different in this regard than are other, less abstract types of constructions investigated so far. This also leads us to hypothesise that the semantics of quantifiers not mentioned here may be an adaptation of language to the invariant features of the world.

It is interesting that, as exemplified by our analysis, investigating the influence of environmental constraints on quantifier meaning requires a parallel, ecologically valid analysis of such influences on lower-order concepts. This methodological caveat follows directly from the fact that the truth value of quantified sentences depends not only on the meaning of quantifier determiners (denoting higher-order concepts), but also on the meaning of nouns and verb phrases (denoting lower-order concepts) (Barwise \& Cooper, 1981).

Communicative pressures are certainly not the only selectional forces that are important for explaining the evolution of meaning. Another important universal pressure stems from our cognitive limitations (Christiansen \& Chater, 2008, 2016b). Recent insights at the intersection of formal semantics and cognitive science show that cognitive constraints influence the verification of quantified sentences (Szymanik, 2016). There are also attempts to explain quantifier semantic universals in terms of learnability pressures (Steinert-Threlkeld \& Szymanik, 2017). It seems that enriching evolutionary explanations of quantifier meaning with plausible cognitive constraints should make the entire picture more accurate. $^{6}$

Finally, it would be beneficial to validate the formal model proposed in the paper on authentic data obtained from samples collected experimentally or from recorded linguistic usage (corpus studies).

\section{Acknowledgements}

The author was supported by the Polish National Science Centre grant number 2015/19/B/HS1/03292.

\footnotetext{
${ }^{6}$ For example, a model by Kalociński, Gierasimczuk, and Mostowski (2015) assumes a tentative simplicity ordering over proportional quantifier meanings based on the size of the denominator, yielding $1 / 2$ as one of the simplest meanings available. See also Kalociński, Mostowski, and Gierasimczuk (2018).
} 


\section{References}

Barwise, J., \& Cooper, R. (1981). Generalized Quantifiers and Natural Language. Linguistics and Philosophy, 4, 159-219.

Christensen, P., Fusaroli, R., \& Tylén, K. (2016). Environmental constraints shaping constituent order in emerging communication systems: Structural iconicity, interactive alignment and conventionalization. Cognition, 146, $67-80$.

Christiansen, M. H., \& Chater, N. (2008). Language as shaped by the brain. Behavioral and Brain Sciences, 31(05), 489-509.

Christiansen, M. H., \& Chater, N. (2016a). Creating Language: Integrating Evolution, Acquisition, and Processing. MIT Press.

Christiansen, M. H., \& Chater, N. (2016b). The Now-or-Never bottleneck: A fundamental constraint on language. Behavioral and Brain Sciences, 39, e62.

Easley, D., \& Kleinberg, J. (2010). Networks, crowds, and markets: Reasoning about a highly connected world. New York: Cambridge University Press.

Hofbauer, J., \& Sigmund, K. (1998). Evolutionary games and population dynamics. Cambridge University Press.

Kalociński, D., Gierasimczuk, N., \& Mostowski, M. (2015). Quantifier Learning: An Agent-based Coordination Model. In Proceedings of the 2015 International Conference on Autonomous Agents and Multiagent Systems (pp. 1853-1854). Richland, SC: International Foundation for Autonomous Agents and Multiagent Systems.

Kalociński, D., Mostowski, M., \& Gierasimczuk, N. (2018). Interactive semantic alignment model: Social influence and local transmission bottleneck. Journal of Logic, Language and Information. (to appear)

Katsos, N., Cummins, C., Ezeizabarrena, M.-J., Gavarró, A., Kuvač Kraljević, J., Hrzica, G., Grohmann, K. K., Skordi, A., López, K. Jensen de, Sundahl, L., Hout, A. van, Hollebrandse, B., Overweg, J., Faber, M., Koert, M. van, Smith, N., Vija, M., Zupping, S., Kunnari, S., Morisseau, T., Rusieshvili, M., Yatsushiro, K., Fengler, A., Varlokosta, S., Konstantzou, K., Farby, S., Guasti, M. T., Vernice, M., Okabe, R., Isobe, M., Crosthwaite, P., Hong, Y., Balčiūnienè, I., Ahmad Nizar, Y. M., Grech, H., Gatt, D., Cheong, W. N., Asbjørnsen, A., Torkildsen, J. v. K., Haman, E., Miękisz, A., Gagarina, N., Puzanova, J., Anđelković, D., Savić, M., Jošić, S., Slančová, D., Kapalková, S., Barberán, T., Özge, D., Hassan, S., Chan, C. Y. H., Okubo, T., Lely, H. van der, Sauerland, U., \& Noveck, I. (2016). Cross-linguistic patterns in the acquisition of quantifiers. Proceedings of the National Academy of Sciences, 113(33), 9244-9249.

Kemp, C., \& Regier, T. (2012). Kinship Categories Across Languages Reflect General Communicative Principles. Science, 336(6084), 1049-1054. 
Levinson, S. C. (1996). Language and space. Annual Review of Anthropology, 25(1), 353-382.

Levinson, S. C. (2003). Space in language and cognition: Explorations in cognitive diversity (Vol. 5). Cambridge University Press.

Lindsey, D. T., \& Brown, A. M. (2002). Color Naming and the Phototoxic Effects of Sunlight on the Eye. Psychological Science, 13(6), 506-512.

Pauw, S., \& Hilferty, J. (2012). The emergence of quantifiers. Experiments in Cultural Language Evolution, 3, 277.

Pauw, S., \& Hilferty, J. (2016). Embodied cognitive semantics for quantification. Belgian Journal of Linguistics, 30(1), 251-264.

Peters, S., \& Westerståhl, D. (2006). Quantifiers in Language and Logic. Oxford: Oxford University Press.

Plewczyński, D., Łukasik, M., Kurdej, K., Zubek, J., Rakowski, F., \& RączaszekLeonardi, J. (2014). Generic Framework for Simulation of Cognitive Systems: A Case Study of Color Category Boundaries. In Man-Machine Interactions 3 (pp. 385-393). Springer.

Steels, L. (1997). Constructing and sharing perceptual distinctions. In M. van Someren \& G. Widmer (Eds.), Machine Learning: ECML-97: 9th European Conference on Machine Learning Prague, Czech Republic, April 23-25, 1997 Proceedings (pp. 4-13). Berlin, Heidelberg: Springer Berlin Heidelberg. (DOI: 10.1007/3-540-62858-4_68)

Steinert-Threlkeld, S., \& Szymanik, J. (2017). Learnability and Semantic Universals. (unpublished manuscript http://semanticsarchive.net/ Archive/mQ2y2y2z/LearnabilitySemanticUniversals. pdf)

Szymanik, J. (2016). Quantifiers and Cognition: Logical and Computational Perspectives (1 ed.). Springer International Publishing.

Szymanik, J., \& Thorne, C. (2017). Exploring the relation between semantic complexity and quantifier distribution in large corpora. Language Sciences, 60, 80-93. 